\title{
Uncertainty propagation in multibody human model dynamics
}

\author{
Antoine Muller ${ }^{* 1,2}$, Charles Pontonnier ${ }^{1,2,3}$, and Georges Dumont ${ }^{1,2}$ \\ ${ }^{1}$ École Normale Supérieure de Rennes, Avenue Robert Schuman, \\ 35170 Bruz \\ ${ }^{2}$ IRISA/INRIA MimeTIC, Campus de Beaulieu, 35042 Rennes \\ Cedex, France \\ ${ }^{3}$ Écoles de Saint-Cyr Coëtquidan, 56380 Guer
}

08 February 2017

\begin{abstract}
In biomechanics, calibration of body segment inertial parameters (BSIP) is crucial to take into account subject morphological specificities. To avoid strenuous protocols, identification methods based on rigid body dynamics laws have been proposed. Thanks to a motion capture system and force platforms, these methods optimize BSIP by minimizing errors in the equations of motion. These errors can be defined as the dynamic residuals reflecting inaccuracies arising from estimated BSIP, as well as from kinematics and force plate measurements. The current study aims at evaluating the part of uncertainty on the dynamic residuals directly related to kinematics and force plate measurements. To answer this question, we captured the movements of 10 participants performing a standardized motion. We then applied a Monte Carlo-based approach to introduce variations in the kinematics and force plate measurements, and evaluated the reconstructed difference on the dynamic residuals. Results show that, first, the BSIP evaluation using a regression method seemed to be an acceptable estimate for the studied subjects. Second, the part of uncertainty in the dynamic residuals was significantly higher than the dynamic residuals obtained. In conclusion, a subject-specific calibration of the BSIP based on dynamic residuals, for this model and protocol, seems irrelevant and prone to overfitting of BSIP.
\end{abstract}

Keywords: Inverse dynamics; Kinematics; Dynamic residuals; Optimization; BSIP; Monte Carlo method

*Corresponding author: antoine.muller@irisa.fr 


\section{Introduction}

Computation of body segment inertial parameters (BSIP) has been shown to be crucial in biomechanical studies $[1,2]$. Specifically, the personalization of mass, position of center of mass (CoM) and inertia of each body part makes inverse dynamics more accurate and realistic. Several methods have therefore been suggested to estimate subject-specific BSIP.

First, regression methods based on anthropometric databases collected from cadaver studies $[3,4]$ or from in vivo body scanning $[5,6,7,8]$ have been proposed to estimate BSIP. These methods aim at scaling BSIP based on subject's height and weight. The use of regression rules is simple and useful to get a generic or average representation of human inertial characteristics. However, such methods, based on averages from a limited sample of subjects, do not take into account subject morphological specificities, e.g. obesity, amputated limb...

A second approach consists in using in vivo geometrical measurements $[9$, $10,11]$. These methods use 3D scanning or magnetic resonance imaging to reconstruct segmental geometries. BSIP are then estimated by assigning density values to the reconstructed volumes. These methods are accurate but expensive and can be invasive (radiations).

More recently, a third family of methods has been proposed to estimate BSIP in vivo. It consists in using motion capture and external force measurements to reproduce the motion dynamics and find the best BSIP that fit with these equations [12]. This problem was solved in two ways. [13] and [14] suggested to write the inverse dynamics problem under a linear form with respect to the inertial parameters. Then, a least squares method solved the optimization problem. Meanwhile, [15] and [16] focused on the 6 degrees of freedom (DoF) joint between the floating-base system and the global reference frame as a measure of the simulation accuracy. The optimization problem consisted in minimizing the generalized forces at this virtual joint, that corresponds to the dynamic residuals.

In their work, [17] stated that "the magnitude of the vector of residuals gives an idea of the accuracy of the simulation, including the kinematic data, the mechanical model and the ground reaction forces' measurements". Thus, errors associated to kinematic data and force platform measurements directly influence optimization results. This may cause overfitting, and distort the BSIP estimates. Indeed, [18] defined overfitting as "asking too much from the available data. Given a certain number of observations in a data set, there is an upper limit to the complexity of the model that can be derived with any acceptable degree of uncertainty". Considering this observation is fundamental and requires a rigorous analysis of the influence of these errors on the dynamic residuals.

Some studies have been conducted to analyze the uncertainties introduced in the inverse dynamics calculus. On one hand, during the walking motion, [15, $19,20]$ analyzed the variation of the resulting joint torques introduced by the uncertainties of different dynamic inputs. On the other hand, [17] assessed different inverse kinematics methods - particularly the effect of the kinematic data consistency [21] — on inverse dynamics consistency. To achieve this, they evaluated inverse dynamics consistency using dynamic residuals.

The current study aims at analyzing the influence of both kinematics and force plate measurements on the dynamic residuals of the equations of motion for a multibody human model. We adopte a Monte Carlo-based approach of 
error propagation from kinematics and force plate measurements to dynamic residuals, presented in the next section. The study was conducted on 10 subjects performing a standardized whole body motion. Results are presented and discussed in Section 3, and a focus is made on the propensity of such residuals to be used as a criterion to optimize the BSIP of such a model.

\section{Materials and methods}

For most biomechanical studies, regression methods are used to estimate BSIP. In identification methods based on optimization, regression approaches are also considered as the initial estimate of the optimization problem. Evaluating the accuracy of regression-based methods in estimating BSIP is therefore important in order to evaluate their validity when used to compute inverse dynamics. Moreover, some studies identify the subject-specific BSIP thanks to the minimization of dynamic residuals. As previously discussed, it then seems important to evaluate if there is a risk of overfitting during the optimization. Therefore the present study proposes to evaluate the following hypotheses:

H1: regression methods compute acceptable body segment inertial parameters (BSIP) estimate for the inverse dynamics problem;

H2: dynamic residuals can be used to achieve a subject-specific BSIP calibration without overfitting.

To answer these questions, movements were performed by a group of subjects. The experimental data were used to drive a multibody human model scaled using a classical regression rule and dynamic residuals were computed and analyzed to investigate hypothesis H1. Then, a Monte Carlo-based method was applied to both kinematics and force plate measurements to determine the uncertainty due to these errors in the dynamic residuals. This approach allowed us to investigate hypothesis $\mathbf{H 2}$.

General notations In this paper, re, pe and $d r$ refer respectively to the reconstruction error, the platform measurement error and the dynamic residuals. Considering $x$ and $y$ as the general notation of these abbreviations, $\epsilon_{x}$ refers to the accuracy of $x$. For inputs of Monte Carlo methods, $\Delta \epsilon_{x}$ indicates the coefficient of dispersion on $x$-perturbed data. For outputs, $\Delta \epsilon_{y}^{x}$ indicates the coefficient of dispersion on $y$ introduced by the $x$-perturbed data.

\subsection{Reference data}

Reference data - data of the analysis pipeline without perturbation - are evaluated using an inverse dynamics framework (Fig. 1) based on the methods described in [28], [29] or [30]. In this study, all reference variables are marked with * . Experimental data used as input of this step are the marker positions $\boldsymbol{X}_{\boldsymbol{e x p}}^{*}$ and ground reaction forces of the two platforms $\boldsymbol{F}_{\boldsymbol{p}}^{*}$ (1) (see the experimental protocol in Section 2.3)

$$
F_{p}^{*}=\left[\begin{array}{l}
F_{p_{1}}^{*} \\
F_{p_{2}}^{*}
\end{array}\right]
$$

The reference data are then computed by the four steps presented below (Fig. 1). First, an inverse kinematics step computes the joint coordinates $\boldsymbol{q}^{*}$. 
Joint torques are then computed in the inverse dynamics step by using the experimental ground reaction forces $\boldsymbol{F}_{\boldsymbol{p}}^{*}$. For the inverse kinematics and inverse dynamics steps, the accuracy of the results are quantified by assessing two indicators: $\epsilon_{r e}^{*}$ and $\epsilon_{\boldsymbol{d r}}^{*}$.

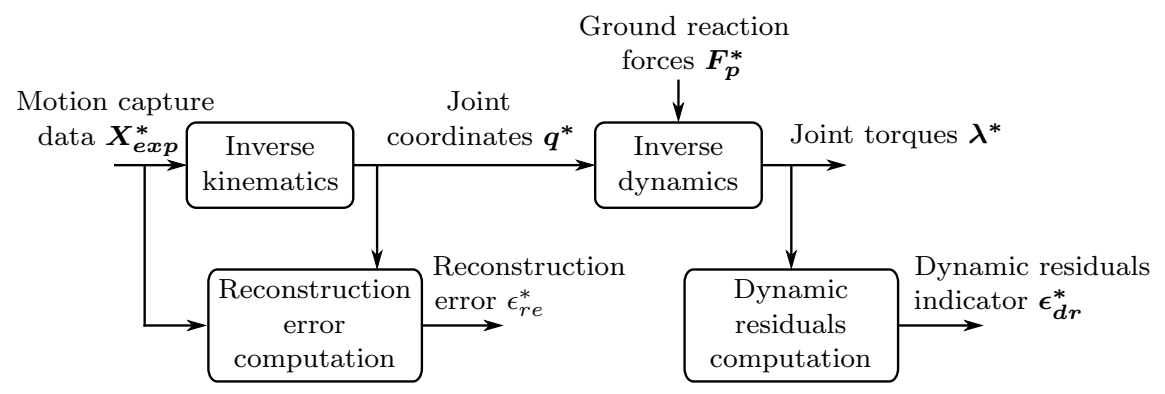

Figure 1: Pipeline of our reference inverse dynamics problem. From the experimental data (motion capture data $\boldsymbol{X}_{\boldsymbol{e x p}}^{*}$ and ground reaction forces $\boldsymbol{F}_{\boldsymbol{p}}^{*}$ ), joint torques $\boldsymbol{\lambda}^{*}$ are computed by the inverse kinematics and the inverse dynamics steps. The accuracy of the results is quantified by assessing two indicators: $\epsilon_{r e}^{*}$ and $\epsilon_{d r}^{*}$

Inverse kinematics The inverse kinematics problem consists in finding the vector of generalized joint coordinates that best reconstructs the movement from the experimental marker positions $\boldsymbol{X}_{\boldsymbol{e x p}}$. For each time step $i$, the sum of squared distances between measured and model-determined marker positions is minimized (2) by using the global optimization method proposed by [31].

$$
\min _{\mathbf{q}(i)} f(\mathbf{q}(i))=\sum_{m=1}^{n_{m}}\left\|\boldsymbol{X}_{\boldsymbol{m o d}, \boldsymbol{m}}(\mathbf{q}(i))-\boldsymbol{X}_{\boldsymbol{e x p}, \boldsymbol{m}}(i)\right\|^{2}
$$

$\mathbf{q}(i)$ is the vector of generalized coordinates at time step $i, \boldsymbol{X}_{\boldsymbol{m o d} \boldsymbol{m} \boldsymbol{m}}(\mathbf{q}(i))$ the coordinates of marker $m$ obtained by forward kinematics, $\boldsymbol{X}_{\boldsymbol{e x p}, \boldsymbol{m}}(i)$ the experimental coordinates of marker $m$. The total number of markers is $n_{m}$.

The joint coordinate trajectories are composed of the set of joint coordinates obtained for each time step. Then, these trajectories are filtered using a 4-th order Butterworth low pass filter with a cut-off frequency of $5 \mathrm{~Hz}$ and no phase shift.

Reconstruction error computation Accuracy of the inverse kinematics step is assessed by computing the reconstruction error $\epsilon_{r e}$. This indicator corresponds to the average distance between real and model markers over the whole recording period, defined as the global reconstruction error in [32]. Finally, the average error for all markers is computed by Equation (3).

$$
\epsilon_{r e}=\frac{1}{n_{m} n_{f}} \sum_{m=1}^{n_{m}} \sum_{i=1}^{n_{f}}\left\|\boldsymbol{X}_{\boldsymbol{m o d}, \boldsymbol{m}}(\mathbf{q}(i))-\boldsymbol{X}_{\boldsymbol{e x p}, \boldsymbol{m}}(i)\right\|
$$

$n_{f}$ is the total number of time steps $i$. 
Inverse dynamics The equation of motion of the system can be written in the general form (4).

$$
\boldsymbol{M}(\boldsymbol{q}) \cdot \ddot{\boldsymbol{q}}+\boldsymbol{C}(\boldsymbol{q}, \dot{\boldsymbol{q}})+\boldsymbol{G}(\boldsymbol{q})+\boldsymbol{\lambda}+\boldsymbol{E}=\mathbf{0}
$$

$\boldsymbol{M}(\boldsymbol{q})$ is the inertia matrix, $\boldsymbol{C}(\boldsymbol{q}, \dot{\boldsymbol{q}})$ is the centrifugal and Coriolis force vector, $\boldsymbol{G}(\boldsymbol{q})$ is the gravity force vector, $\boldsymbol{\lambda}$ is the generalized internal force vector and $\boldsymbol{E}$ is the generalized external force vector. Ground reaction forces $\boldsymbol{F}_{p}^{*}$ are considered as external forces, included in $\boldsymbol{E}$. A recursive Newton-Euler algorithm [24] is used to solve (4), yielding the vector of generalized internal forces $\boldsymbol{\lambda}$.

Dynamic residuals computation Equation (4) can be developed as a floatingbase dynamics equation (5). The upper part of the equation describes the 6 DoF motion of the base (e.g. position and orientation of the pelvis). The lower part describes the motion of all the bodies in the model.

$$
\left[\begin{array}{ll}
M_{11}(q) & M_{1 *}(q) \\
M_{* 1}(q) & M_{* *}(q)
\end{array}\right] \cdot\left[\begin{array}{l}
\ddot{q}_{1} \\
\ddot{q}_{*}
\end{array}\right]+\left[\begin{array}{l}
C_{1}(q, \dot{q}) \\
C_{*}(q, \dot{q})
\end{array}\right]+\left[\begin{array}{l}
G_{1}(q) \\
G_{*}(q)
\end{array}\right]+\left[\begin{array}{c}
\lambda_{1} \\
\lambda_{*}
\end{array}\right]+\left[\begin{array}{l}
E_{1} \\
E_{*}
\end{array}\right]=0
$$

The subscripts 1 and $*$ refer, as proposed in [24], respectively to the first joint (6 DoF virtual joint) and to all the remaining joints. So $\boldsymbol{\lambda}_{\mathbf{1}}$ corresponds to the vector of generalized forces at the 6 DoF joint. It contains 6 components: 3 forces and 3 torques along the 3 -axis of the global reference frame (6).

$$
\boldsymbol{\lambda}_{\mathbf{1}}=\left[\begin{array}{llllll}
F_{x} & F_{y} & F_{z} & M_{x} & M_{y} & M_{z}
\end{array}\right]^{T}
$$

Superscript $T$ means transpose. When performing a motion, $F_{x}$ is the anteroposterior force residual, $F_{y}$ is the lateral-medial force residual, $F_{z}$ is the inferiorsuperior force residual, $M_{x}$ is the abduction moment residual, $M_{y}$ is the flexion moment residual and $M_{z}$ is the internal rotation moment residual.

If the inverse dynamics problem is perfectly solved, all the components of $\boldsymbol{\lambda}_{\mathbf{1}}$ would be null. This property is then used to define the following indicator of the accuracy of the inverse dynamics step: the dynamic residuals indicator $\boldsymbol{\epsilon}_{\boldsymbol{d} \boldsymbol{r}}$, defined as the root-mean-square (RMS) of $\boldsymbol{\lambda}_{\mathbf{1}}$ throughout the motion (7). To allow for inter-subject comparisons, the three effort components of $\boldsymbol{\epsilon}_{\boldsymbol{d r}}$ are normalized by the body weight of the subject $\left(m_{s} . g\right)$ and the three torque components are normalized by the body weight times the height $\left(m_{s} . g . h_{s}\right)$ [15].

$$
\begin{aligned}
& \boldsymbol{\epsilon}_{\boldsymbol{d} \boldsymbol{r}}=\frac{1}{m_{s} \cdot g} \sqrt{\frac{1}{n_{f}}}\left[\sqrt{\sum_{i=1}^{n_{f}}\left(F_{x}(i)\right)^{2}} \sqrt{\sum_{i=1}^{n_{f}}\left(F_{y}(i)\right)^{2}} \sqrt{\sum_{i=1}^{n_{f}}\left(F_{z}(i)\right)^{2}}\right. \\
& \left.\frac{1}{h_{s}} \sqrt{\sum_{i=1}^{n_{f}}\left(M_{x}(i)\right)^{2}} \frac{1}{h_{s}} \sqrt{\sum_{i=1}^{n_{f}}\left(M_{y}(i)\right)^{2}} \frac{1}{h_{s}} \sqrt{\sum_{i=1}^{n_{f}}\left(M_{z}(i)\right)^{2}}\right]^{T}
\end{aligned}
$$




\subsection{Perturbed data}

To identify the uncertainty introduced by the kinematic data and the force plate measurements on the dynamic residuals, we perform a Monte Carlo-based approach, by running Monte Carlo samplings separately on both sources of uncertainty. In Monte Carlo methods, the reference data are perturbed to obtain a set of perturbed samples. For each sample, a known perturbation is applied by adding a noise following a probability distribution. The perturbed inputs are processed through the analysis pipeline. Then, we analyze the distribution of the output error. The following two sections feature the differences in the methods applied for each source of uncertainty.

Reconstruction error First, we aim at determining the influence of the uncertainty introduced by the kinematic data on the dynamic residuals. To apply a Monte Carlo method, a set of perturbed motions in which the reconstruction error follows a known distribution is required. Since reconstruction error is a result of the inverse kinematics step, it can not be directly perturbed. Therefore, we apply perturbations to the reference joint coordinates to generate a database of perturbed motions corresponding to a specified reconstruction error. From a subset of this database, which follows a known distribution, and by fixing the reference ground reaction forces, the distribution of the dynamic residuals is estimated thanks to the inverse dynamics step (Fig. 2). This step corresponds to the inverse dynamics step in the reference pipeline (right part of Fig. 1) with perturbed kinematic data.

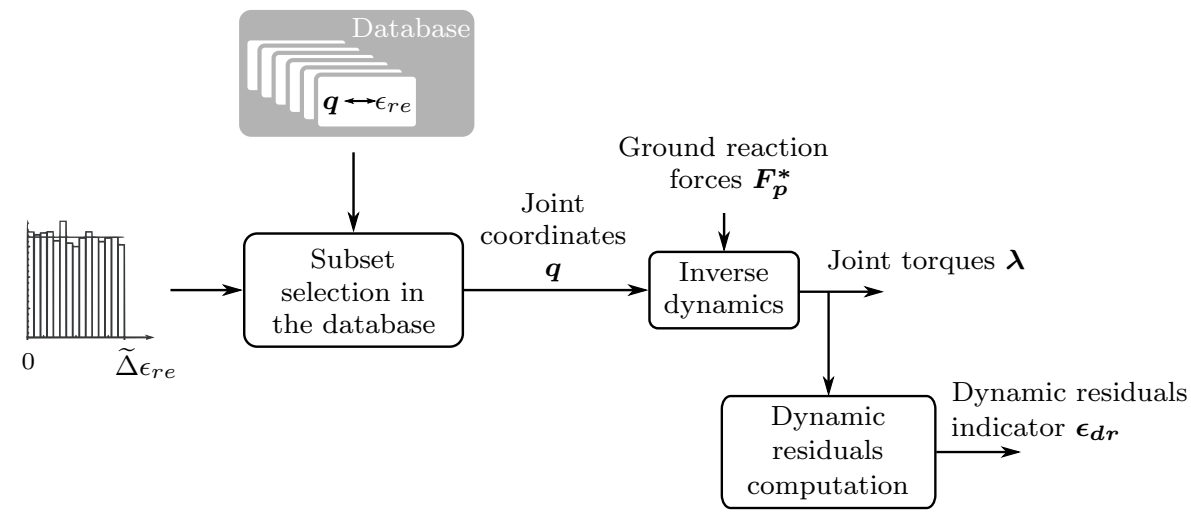

Figure 2: Pipeline used to evaluate the propagation of the uncertainties introduced by the reconstruction error in the dynamic residuals. It corresponds to the inverse dynamics reference pipeline in Figure 1, using perturbed motions

To distort the reference motion, for each time step $i$, a Gaussian noise is added on the reference joint coordinates $\boldsymbol{q}^{*}(8)$ to generate all the perturbed sample $N$ (Fig. 3).

$$
\forall i \in \llbracket 0, n_{f} \rrbracket, \boldsymbol{q}^{\boldsymbol{N}}(i)=\boldsymbol{q}^{*}(i)+\mathcal{N}_{\boldsymbol{n}_{\boldsymbol{q}}}\left(0, \sigma_{q}^{N}\right)
$$

$n_{q}$ is the total number of joints, $\boldsymbol{q}^{\boldsymbol{N}}$ is the vector of generalized coordinates at frame $i$ for the sample $N$ and $\mathcal{N}_{n}(\mu, \sigma)$ is a $n$-dimensional vector with random 
variables normally distributed with mean $\mu$ and variance $\sigma$. For each sample, $\sigma_{q}^{N}$ is randomly selected over the interval $\left[0, \sigma_{q \max }\right]$. The value of $\sigma_{q \max }$ is arbitrarily chosen to allow the maximal value of reconstruction error to be higher than the maximal value needed for the subset selection.

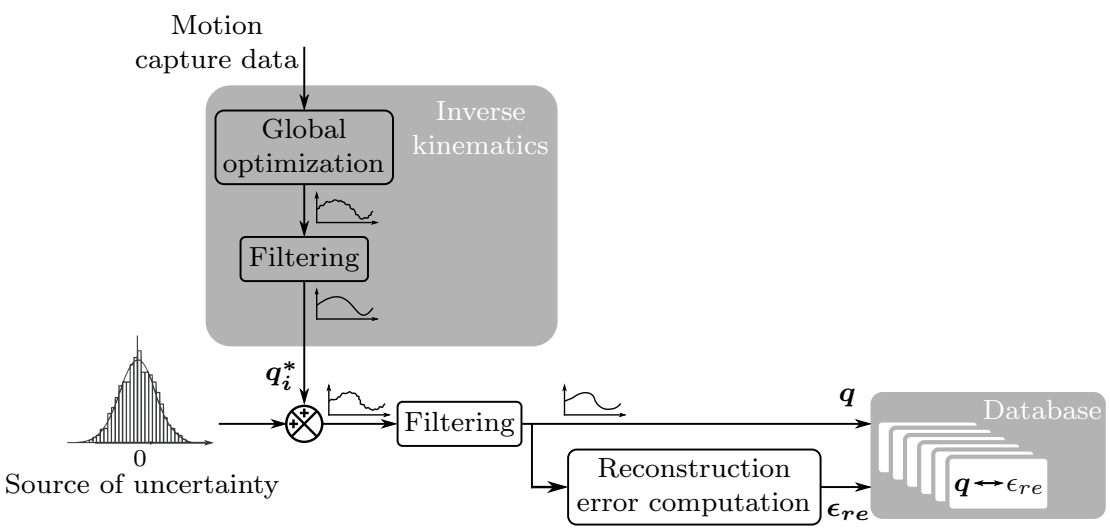

Figure 3: Representation of the method used to introduce noise to the inverse kinematics data. This method is used to generate a database which contains a set of perturbed motions linked to their reconstruction error

Perturbing kinematics data aims at reproducing uncertainties introduced by the motion capture and global optimization steps. To consider these data as an uncertainty, we apply the exact same processing pipeline used for the reference data construction (Section 2.1), which avoids very large accelerations nonexistent in the standard processing. Thus, joint coordinates trajectories are filtered using a 4-th order Butterworth low pass filter with a cut-off frequency of $5 \mathrm{~Hz}$ and no phase shift and finally gathered in a database as shown in Fig. 3.

As the reference reconstruction error $\epsilon_{r e}^{*}$ is the minimal error that can be achieved, we base the subset distribution from this value. We choose a reconstruction error distribution of the subset following a uniform distribution over the interval $\left[\epsilon_{r e}^{*}, \epsilon_{r e}^{*}+\Delta \epsilon_{r e}\right]\left(\Delta \epsilon_{r e}>0\right)$. This distribution is reformulated in the range $\left[0, \widetilde{\Delta} \epsilon_{r e}\right]$, with $\widetilde{\Delta} \epsilon_{r e}$ being the maximal relative reconstruction error added (9).

$$
\widetilde{\Delta} \epsilon_{r e}=\frac{\Delta \epsilon_{r e}}{\epsilon_{r e}^{*}}
$$

In order to constitute a subset of perturbed motions where reconstruction errors follow the desired distribution law, we perform a random selection with replacement on the database. It is conducted according to the previously defined uniform distribution.

The dynamic residuals indicator $\boldsymbol{\epsilon}_{\boldsymbol{d} \boldsymbol{r}}$ is then computed for each perturbed motion in the subset using the reference ground reaction forces. Since the distribution of the input (distribution of the subset reconstruction error) is uniform, the dispersion coefficient should be associated with the range $\Delta \epsilon_{r e}$. As some outliers may appear, the dispersion coefficients of the dynamic residuals indicators $\Delta \boldsymbol{\epsilon}_{\boldsymbol{d} \boldsymbol{r}}^{\boldsymbol{r} \boldsymbol{e}}$ are not directly computed with the maximum and the minimum values. Instead, standard deviations $\boldsymbol{\sigma}$ are computed and $\boldsymbol{\Delta} \boldsymbol{\epsilon}_{\boldsymbol{d} \boldsymbol{r}}^{\boldsymbol{r}}$ is defined in 
agreement with the range of a uniform distribution (10).

$$
\Delta \epsilon_{d r}^{r e}=\sqrt{12} \sigma
$$

The study we conducted (see Results in Section 3) is performed with different values of $\widetilde{\Delta} \epsilon_{r e}$ to analyze the influence of the noise added on the dynamic residuals. To ensure the objective, the maximum relative reconstruction error added is chosen to add once the reference reconstruction error. Thus, the method is repeated five times with $\widetilde{\Delta} \epsilon_{r e}=\{0.2,0.4,0.6,0.8,1\}$.

Force plate measurement error Similarly, to assess the influence of the uncertainty introduced by the force plate measurements on dynamic residuals, we use a set of perturbed force plate data generated by adding a Gaussian noise. Inverse dynamics is then computed using both reference motion data and perturbed force plate data to evaluate dynamic residuals (Fig. 4). This pipeline corresponds to the inverse dynamics step in the reference pipeline (right part in Fig. 1), but using perturbed force plate data.

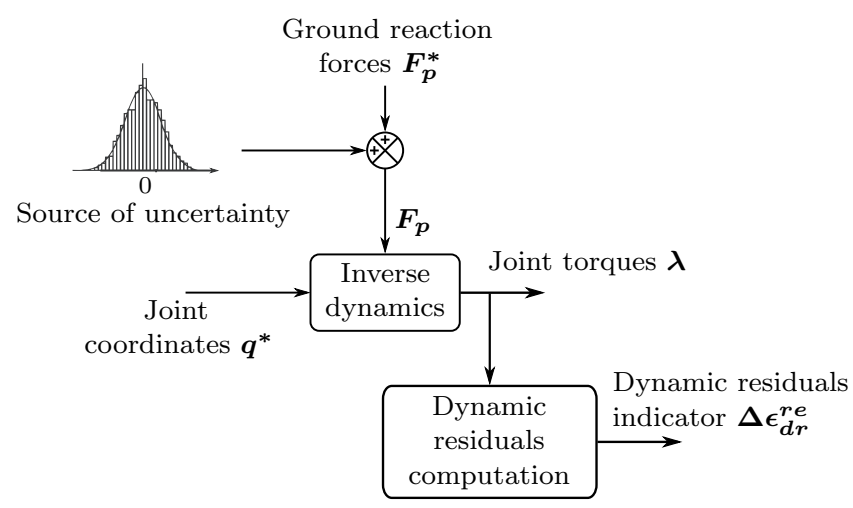

Figure 4: Pipeline evaluating the effect of the force plate data measurement uncertainties on te dynamic residuals. This corresponds to the inverse dynamics reference pipeline in Figure 1, using perturbed force plate data

In the AMTI specifications (see the reference of the used force plates in Section 2.3), force sensitivities are reported to be less than $0.1 \%$ of the applied load, with a minimum of $0,22 \mathrm{~N}$. The accuracy of the center of pressure $(\mathrm{CoP})$ position estimation is reported to be less than $0.2 \mathrm{~mm}$.

Thus, we apply two different Gaussian noise distributions respectively to the forces and the CoP positions to generate all the perturbed samples $N$ (11).

$$
\forall i \in \llbracket 0, n_{f} \rrbracket,\left\{\begin{aligned}
\boldsymbol{C o P ^ { N }}(i) & =\boldsymbol{C o P}^{*}(i)+\mathcal{N}_{\mathbf{3}}\left(0, \sigma_{C o P}\right) \\
\boldsymbol{F}_{\boldsymbol{P}_{\mathbf{1}}}^{\boldsymbol{N}}(i) & =\boldsymbol{F}_{\boldsymbol{P}_{\mathbf{1}}}^{*}(i)+\mathcal{N}_{\mathbf{3}}\left(0, \sigma_{F_{P}}\right) \\
\boldsymbol{F}_{\boldsymbol{P}_{\mathbf{2}}}^{\boldsymbol{N}}(i) & =\boldsymbol{F}_{\boldsymbol{P}_{\mathbf{2}}}^{*}(i)+\mathcal{N}_{\mathbf{3}}\left(0, \sigma_{F_{P}}\right)
\end{aligned}\right.
$$

$\boldsymbol{C o P}^{\boldsymbol{N}}(i)$ is the CoP position vector at time step $i$ for the sample $N, \boldsymbol{F}_{\boldsymbol{P}_{\mathbf{1}}}^{\boldsymbol{N}}(i)$ and $\boldsymbol{F}_{\boldsymbol{P}_{\mathbf{2}}}^{\boldsymbol{N}}(i)$ are the ground reaction force vectors for the sample $N$ on platforms 1 and 2 respectively and $\sigma_{C o P}$ and $\sigma_{F_{P}}$ are the variances of the Gaussian distribution for the CoP positions and for the forces respectively. The values of $\sigma_{C o P}$ 
and $\sigma_{F_{P}}$ are chosen according to the force plate datasheet. The uncertainties, corresponding to the dispersion coefficients, will then be evaluated using the 3 -sigma values.

We generate a set of perturbed force plate data, and compute the dynamic residuals indicator $\boldsymbol{\epsilon}_{\boldsymbol{d} r}$ for each sample. In the same way as in the force plate data, the dispersion coefficients on the dynamic residuals indicators are associated to the 3 -sigma values: $\Delta \epsilon_{d r}^{p e}$.

\subsection{Experimental protocol and biomechanical model}

We applied the method previously described on experimental data. For this purpose, 10 male participants (mean age \pm std: $27.5 \pm 5$ years old, height: $180 \pm 7 \mathrm{~cm}$, mass: $74.1 \pm 7.3 \mathrm{~kg}$ ) participated in this study. All subjects signed an informed consent form before participation. An anonymization protocol was followed for data safeguarding. The experimental setup was composed of 47 motion capture markers placed on standardized anatomical landmarks as recommended by the International Society of Biomechanics (ISB) [22, 23]. Motion capture markers were recorded using a Vicon ${ }^{\circledR}$ motion capture system with 16 cameras (100 $\mathrm{Hz})$. Subjects stood over two AMTI ${ }^{\circledR} 120 \times 60 \mathrm{~cm}$ force platforms $(1000 \mathrm{~Hz})$, each supporting one foot. Each subject visualized a video displaying an avatar performing a standardized movement and imitated the displayed movements (Fig. 5). These movements were designed to activate sequentially each degree of freedom of the kinematical model used thereafter.

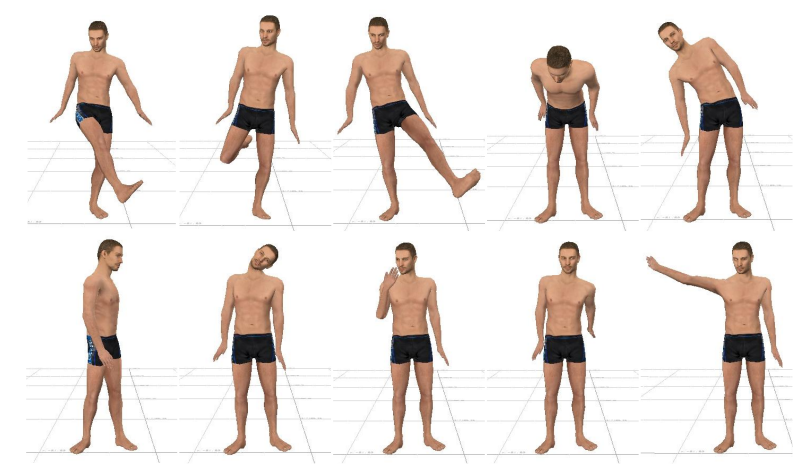

Figure 5: Different postures of the video guide. The video was generated using an avatar animated from motion capture data

The whole body model used in this study is composed of 16 rigid segments linked by 15 joints and exhibits 35 degrees of freedom (Fig. 6). A virtual 6DoF joint connects the pelvis to the global reference frame, allowing to convert a floating-base system into an equivalent fixed base system [24]. To simplify the inverse kinematics and inverse dynamics steps, the model is hierarchically described, allowing the use of recursive functions [25]. Conventions for global and local reference frame and for rotation combinations follow ISB recommendations [26].

A preliminary subject-specific kinematical calibration was performed using motion capture data [27]. Using a set of postures, limb lengths and model marker positions were optimized to best fit captured marker positions. From 


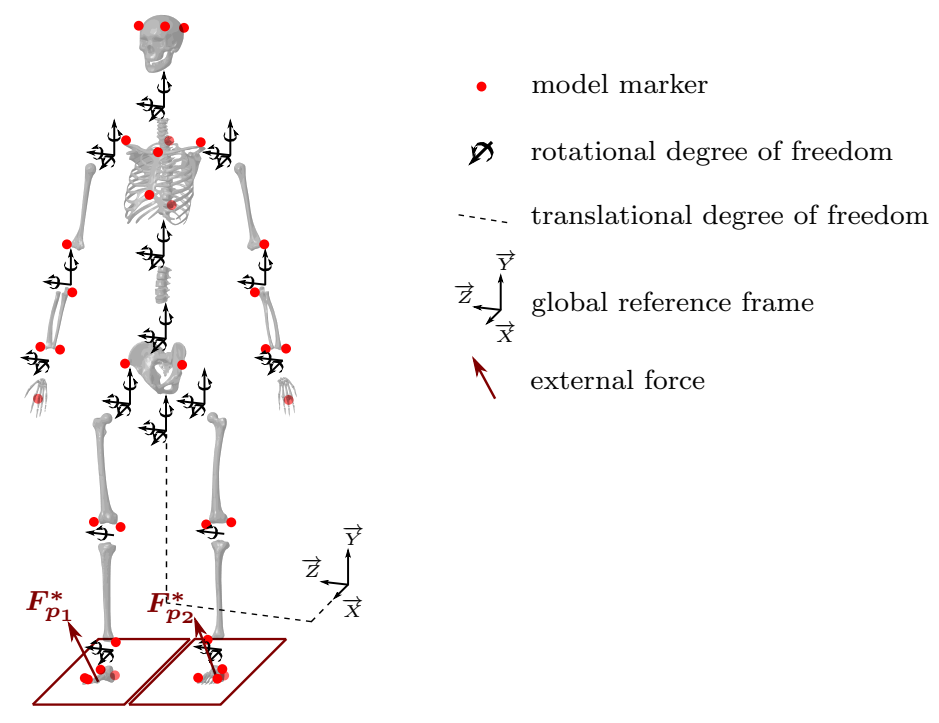

Figure 6: Kinematic model, model marker position and external forces applied to the biomechanical model (bones graphics are adapted from the AnyBody Managed Repository: http://www.anybodytech.com/)

this calibrated model, BSIP were then estimated using the regression method proposed by [5]. This regression method, widely used in human multibody dynamics analyses, is the one evaluated with respect to hypothesis $\mathbf{H 1}$.

\section{Results and discussion}

The present study analyzes the uncertainties propagation in the dynamic residuals. Such a study is suitable to assess the relevance of a BSIP calibration achieved by dynamic residuals minimization. In the following sections, we will analyze separately the influence of the different sources of errors using a Monte Carlo-based approach (Section 3.1 and 3.2). We will then evaluate the part of uncertainty introduced by these errors in the dynamic residuals values (Section $3.3)$.

\subsection{Reconstruction error}

We first computed the dispersion coefficients obtained from reconstruction error propagation (crosses in Fig. 7, where each subject is represented by a distinctive color). To compare results between subjects, the different coefficients are displayed according to the range of the uniform distribution $\Delta \epsilon_{r e}$. The reference reconstruction error being different for each subject, the abscissa values of $\Delta \epsilon_{r e}$ are different among subjects.

For each component of the dynamic residuals, the propagation of the reconstruction error in the dynamics seems to be linear. To characterize it, we perform a linear regression using a least-squares method on the results of each subject (straight lines in Fig. 7). The regression function is constrained to have an intercept at zero. This corresponds to a zero coefficient of dispersion with no 

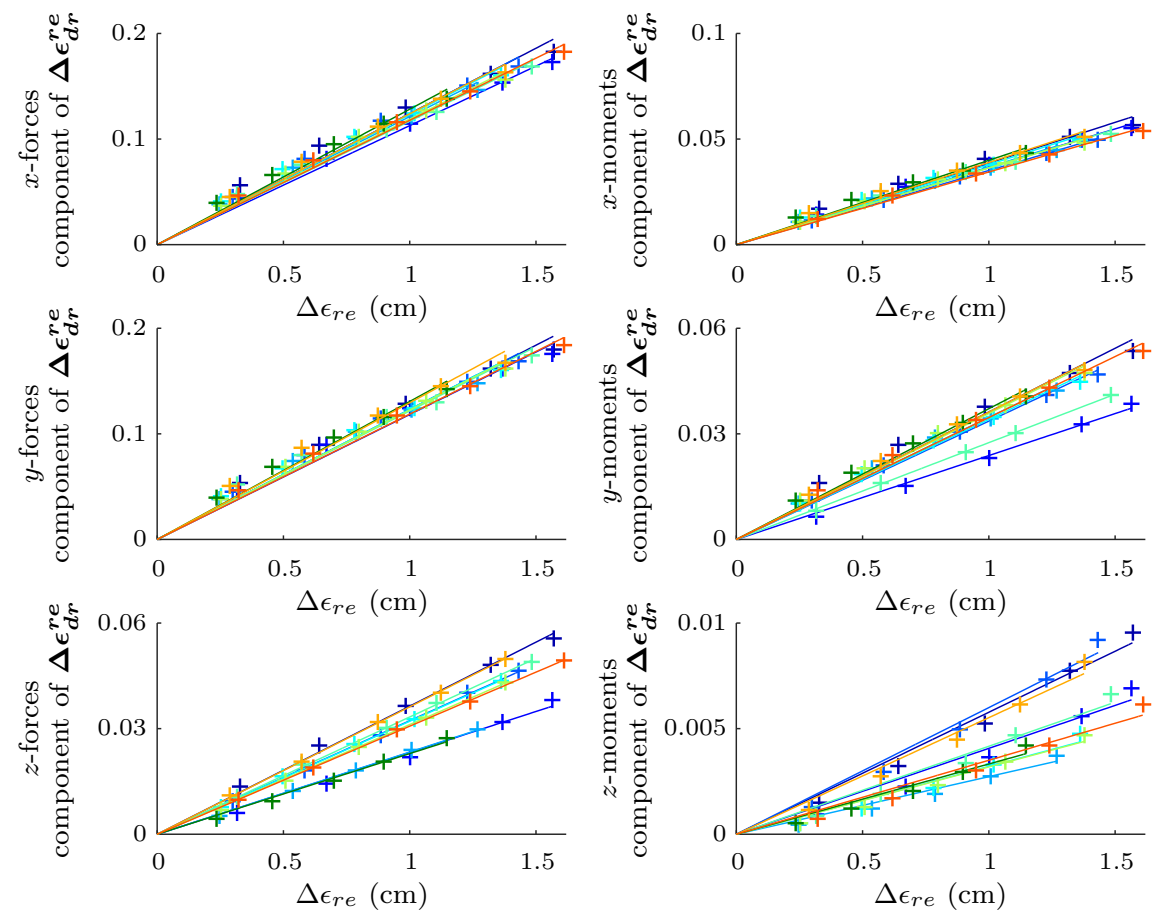

Figure 7: Components of the dispersion coefficients $\Delta \epsilon_{d r}^{r e}$ of the dynamic residuals indicators according to the range of the uniform distribution $\Delta \epsilon_{r e}$. The 3 effort components of these residuals are normalized by the weight of the subject and the 3 torque components are normalized by the body weight times the height

more reconstruction error added. The linear regressions result in a 6-coefficient slope vector $\boldsymbol{m}_{\Delta \epsilon}^{r e}$ for each subject. Furthermore, the R-squared value is always within the [0.963 0.999] range.

From this result, we can assume that the uncertainty due to the reconstruction error corresponds to the dispersion coefficients obtained with $\Delta \epsilon_{r e}=\epsilon_{r e}^{*}$ $\left(\widetilde{\Delta} \epsilon_{r e}=1\right)$. Therefore, a priori knowledge of $\boldsymbol{m}_{\Delta \epsilon}^{r e}$ for a given subject enables the computation of the uncertainty due to the kinematics only based on the reference reconstruction error. As the reference reconstruction error depends largely on the motion, we compare the consistency between subjects by using $m_{\Delta \epsilon}^{r e}$.

Fig. 8 shows, for each dynamic residuals component, the repartition of the different slopes obtained by including the results of all the subjects. These boxplots show that, for each component, the slopes are consistent while the reference reconstruction errors are slightly different.

Moreover, the slope values are different according to the considered axis. For instance, the uncertainty due to reconstruction error is smaller on the vertical axis (z-axis) for both force and moment components. For the force component on the $z$-axis, the relative influence of kinematics is relatively lower compared to the weight which is influential in this component. Because the inertia of 


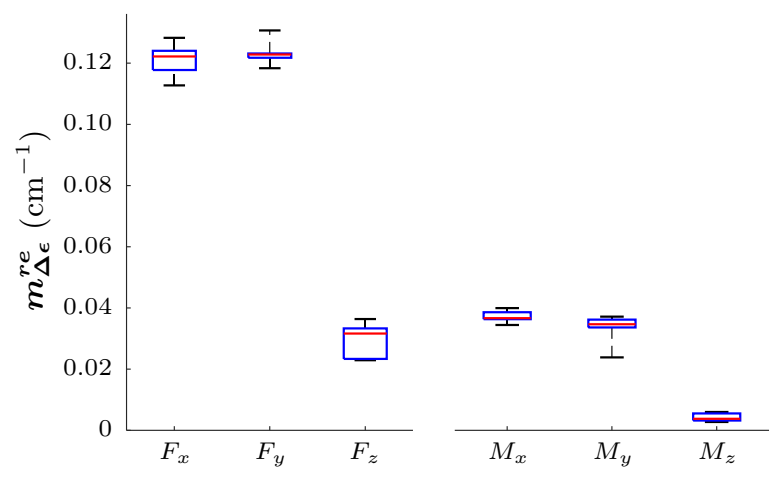

Figure 8: Boxplots representing the slopes of the linear regressions which evaluate dispersion coefficients on the dynamic residuals indicators according to $\Delta \epsilon_{\text {re }}$

whole body is lower around the vertical axis, the influence of kinematics is less important on the $z$-axis moment component. Moreover, the results obtained with the $x$-axis and with the $y$-axis are of the same order of magnitude. This is probably due to the fact that the performed motion did not occur in a specified direction unlike, for example, walking motions.

\subsection{Force plate measurement error}

For each subject, we performed a statistical analysis of the repartition of the dynamic residuals indicators after the propagation of the force plate data measurement uncertainties. Fig. 9 represents the resulting dispersion coefficients. The magnitude of the results seems to be consistent for every subject.

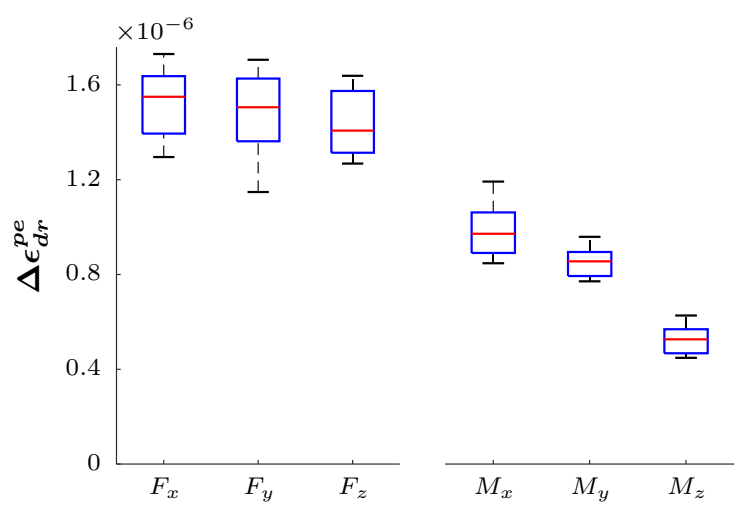

Figure 9: Boxplots representing the dispersion coefficients in the dynamic residuals indicators after the propagation of the force plate data measurement uncertainties. The 3 effort components are normalized by the weight of the subject and the 3 torque components are normalized by the body weight times the height 


\subsection{Comparison with the reference dynamic residuals}

One of the purposes of the study was to evaluate the possibility of refining BSIP based on dynamic residuals minimization without overfitting (H2). Therefore, we compare the reference dynamic residuals to the uncertainties introduced by the reconstruction error and by the force plate measurements. The global uncertainties $\boldsymbol{\Delta} \boldsymbol{\epsilon}_{\boldsymbol{d} \boldsymbol{r}}$ (12) are obtained from the different uncertainties computed separately (Sections 3.1 and 3.2). The first part of (12) corresponds to the uncertainties due to the reconstruction error and the second part corresponds to the uncertainties due to the force plate data measurement.

$$
\Delta \epsilon_{d r}=\epsilon_{r e}^{*} \cdot m_{\Delta \epsilon}^{r e}+\Delta \epsilon_{d r}^{p e}
$$

First of all, it is easy to see that the part of uncertainty due to the force plates measurement was significantly lower than the one due to the reconstruction error (5 orders of magnitude lower). This source of uncertainty can therefore be neglected in this study. Fig. 10 shows the reference dynamic residuals along with the uncertainties in the dynamic residuals for each subject.

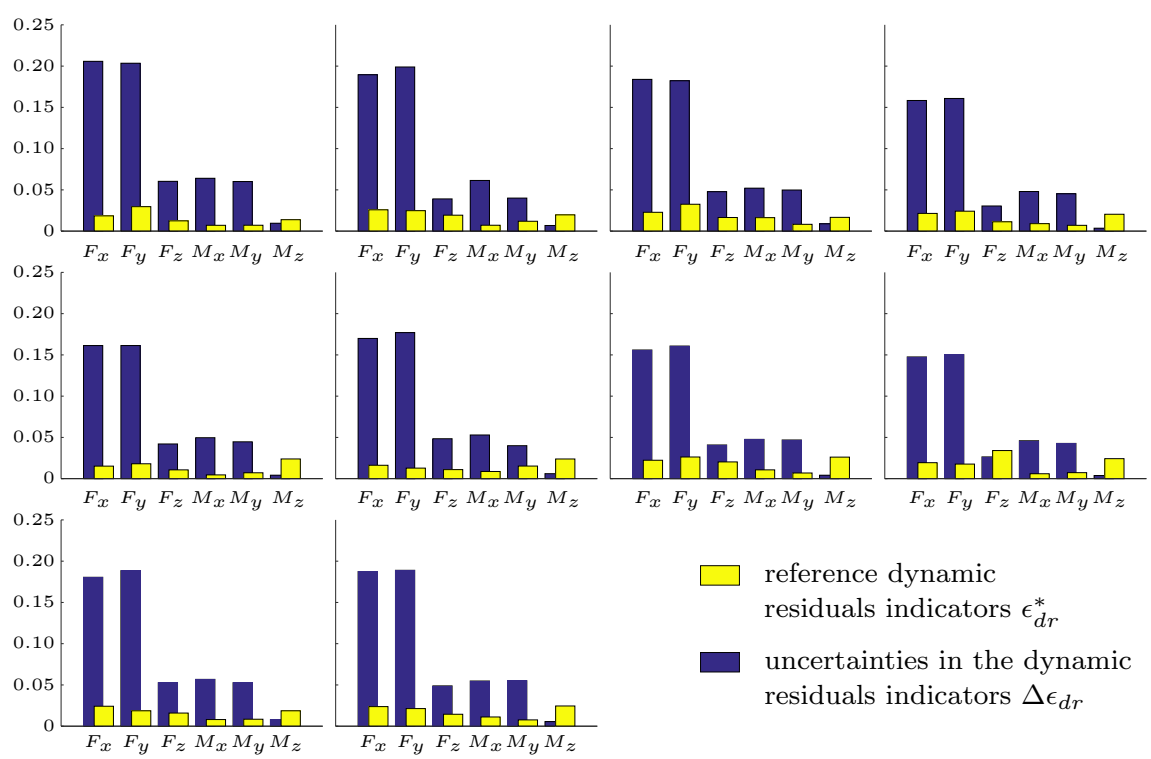

Figure 10: Reference dynamic residuals indicators and global uncertainties in the dynamic residuals indicators for each component. Each graph represents the results with one subject

For all subjects, the mean value and the standard deviation (mean(std)) of the reference force components dynamic residuals indicators is $2 \%(0.6 \%)$ (percentage of the weight of the subject) and for the reference torque components is $1.3 \%(0.7 \%)$ (percentage of the weight times the height). These orders of magnitude of inverse dynamics inconsistency are similar to the values obtained by [14] after a subject-specific BSIP calibration. These results seem to indicate that the regression-based BSIP evaluation is an acceptable estimate to calibrate the model, fully supporting hypothesis H1. This might be attributed to the fact that the volunteers were regular people (healthy, no high-level athletes, neither 
underweight nor obese: Body Mass Index within [18.5 30] range). So we can suppose that, for these subjects, the anthropometric data of [5] were close to the subject-specific parameters.

Overall, the uncertainties in the dynamic residuals are higher than the reference dynamic residuals, except for the vertical moment component ( $z$-axis). However, for this component, the difference between the global uncertainty and the reference value is low compared to the other differences.

The study shows that, in the computation of dynamic residuals, uncertainties introduced by kinematic data and by force plate measurements are too high to use these residuals to achieve a subject-specific BSIP calibration. Indeed, the residuals are here widely explained by the reconstruction error and minimizing them to achieve a BSIP calibration would lead to erroneous results. In conclusion, with regard to those results, the experimental protocol and the multibody model used, hypothesis $\mathbf{H 2}$ is refuted. This result partly contradicts some recent studies about BSIP calibration [14, 16]. Some lines of approach are proposed below to explain these contradictions.

First, we showed that reference dynamic residuals in our study were similar those obtained by [14] after a subject-specific BSIP calibration. That means that the inconsistency of the inverse dynamics problem is small. This makes it difficult to use this inconsistency to achieve a subject-specific BSIP calibration. Therefore, the method would meet a more competitive challenge with nonregular people, for example high-level athletes or amputees.

Moreover, our study showed that uncertainties introduced by the kinematic data explain most of the dynamic residuals. To reduce the influence of the kinematic errors on dynamic results, [33] and [34] proposed a global resolution of the inverse dynamics (least-squares method). Unlike the recursive NewtonEuler algorithm which propagates the errors along the kinematical chain, the least-squares method determines joint torques that better respect the global dynamic balance. By considering all the time steps simultaneously, [33] also proposed to eliminate constant bias as a wrong BSIP estimation. However, even for only one time step, using this method requires the implementation and the resolution of a large and complex system of equations, for which it is often difficult to find a solution and for which an initial guess is required to start the optimization. This problem is then increased tenfold by considering all the time steps simultaneously. This is why such a method asks for further developments to be fully usable as a solution to the BSIP estimation problem.

Finally, the kinematic model used in the current study was relatively simple. For example the complex structure of the shoulder was here modeled as a spherical joint which does not allow to take into account the motion of the clavicle or the sliding of the scapula. Thus, the reference reconstruction error - average of $1.4 \mathrm{~cm}$ - is more important than data available in the literature [35] for the lower part of the body. This can have two different impacts on the performed study.

On one side, the reconstruction error introduced by the use of this model could directly introduce dynamic residuals. Complementary studies could be explored: a first approach could consist in adaptively modifying the kinematical model depending on the most important reconstruction error. This could for instance take into account the complex closed-loop geometry of the shoulder [36] or a detailed lumbar spine model [37]. A second approach could be to select only a set of motion sequences to perform BSIP calibration, by selecting only 
sequences where the uncertainties introduced by the reconstruction error would be sufficiently low to achieve a subject-specific BSIP calibration. Acting on the same principle, [38] suggested a method to select sequences of motion with optimal excitation properties. These two methods could be then combined.

On the other side, the reconstruction error introduced by the use of this model could introduce an additional uncertainty without adding dynamic residuals. This seems the case here because, despite the important reconstruction error, reference dynamic residuals are similar to those obtained with a subjectspecific calibrated model [14]. However, the model could be improved by better understanding uncertainties introduced by kinematic data. For this end, we can use soft tissue artefacts (STA) models [39]. These artifacts have been recognized to be the major source of error in human motion analysis [40]. The reconstruction error could also be computed by weighting differently each marker, unlike the global indicator used in this study. As [17] proposed to assign weights to each marker during the global optimization method - according to STA related to the considered segment - , weights may reflect the impact of the marker reconstruction error on dynamics. Indeed, the impact of marker reconstruction error on dynamic residuals is influenced by its position in the kinematic model. For example, the reconstruction error of markers of the hand (distal segment with low mass) has probably a lower impact on the dynamics than the reconstruction error of markers of the trunk. Moreover, a segment with several markers has a stronger impact on the reconstruction error computation whereas its impact on the dynamics is not necessarily dominant. In this manner, we could use a Monte Carlo approach to add noise on each marker, proportionally to its reference reconstruction error. This would probably add some noise close to the characteristics of the reference reconstruction error.

\section{Conclusion}

In this paper, we proposed a method to quantify the uncertainties propagation in the dynamics of multibody human models. In particular, we assessed possibility of minimizing dynamic residuals to calibrate BSIP of the model without overfitting.

In addition to BSIP, two sources of uncertainties are propagated in the inverse dynamics problem: kinematic data and force plate data. We performed a Monte Carlo-based approach, by running Monte Carlo samplings separately on both sources of uncertainty to assess their propagation their propagation in the dynamic residuals. The global uncertainty introduced was then compared to the dynamic residuals obtained with the reference pipeline.

Our study allowed to assess two hypotheses presented at the beginning of this paper. First, estimating BSIP with regression methods seems to provide an acceptable subject-specific approximation, as hypothesized in H1. Second, the introduced uncertainties were significantly higher than the reference dynamic residuals. Therefore, using dynamic residuals for subject-specific BSIP calibration does not guarantee that overfitting can be avoided, thus not supporting hypothesis H2. Some lines of approach were proposed to explain these results and to improve the uncertainty propagation understanding in multibody human model dynamics. 


\section{Acknowledgements}

The authors wish to thank Ana Lucia Cruz Ruiz, Diane Haering, Ludovic Hoyet and Anthony Sorel for their kind support. This study was partially funded by the ANR project ENTRACTE (Grant agreement: ANR 13-CORD-002-01).

\section{References}

[1] Andrews, J.G., Mish, S.P.: Methods for investigating the sensitivity of joint resultants to body segment parameter variations. Journal of Biomechanics 29(5), 651-654 (1996)

[2] Rao, G., Amarantini, D., Berton, E., Favier, D.: Influence of body segments' parameters estimation models on inverse dynamics solutions during gait. Journal of Biomechanics 39(8), 1531-6 (2006)

[3] Chandler, R.F., Clauser, C.E., McConville, J.T., Reynolds, H.M., Young, J.W.: Investigation of inertial properties of the human body. Tech. Rep. March (1975)

[4] Dempster, W.: Space requirements of the seated operator: geometrical, kinematic, and mechanical aspects of the body, with special reference to the limbs (1955)

[5] Dumas, R., Chèze, L., Verriest, J.: Adjustments to McConville et al. and Young et al. body segment inertial parameters. Journal of biomechanics 40(3), 543-53 (2007)

[6] Leva, P.D.: Adjustments to Zatsiorsky-Seluyanov's segment inertia parameters. Journal of biomechanics 29(9), 1223-1230 (1996)

[7] Zatsiorsky, M., Seluyanov, V.: The mass and inertia characteristics of the main segments of the human body. Biomechanics viii-b 56(2), 1152-1159 (1983)

[8] Zatsiorsky, M., Seluyanov, V., Chugunova, L.: In vivo body segment inertial parameters determination using a gamme-scanner method. Biomechanics of human movement: Applications in rehabilitation, sports and ergonomics pp. 196-202 (1990)

[9] Jensen, R.K.: Estimation of the biomechanical properties of three body types using a photogrammetric method. Journal of Biomechanics 11(8), 349-358 (1978)

[10] Pearsall, D.J., Reid, J.G., Ross, R.: Inertial properties of the human trunk of males determined from magnetic resonance imaging. Annals of Biomedical Engineering 22(6), 692-706 (1994)

[11] Davidson, P.L., Wilson, S.J., Wilson, B.D., Chalmers, D.J.: Estimating subject-specific body segment parameters using a 3-dimensional modeller program. Journal of Biomechanics 41(16), 3506-3510 (2008) 
[12] Vaughan, C.L., Andrews, J.G., Hay, J.G.: Selection of body segment parameters by optimization methods. Journal of biomechanical engineering 104(1), 38-44 (1982)

[13] Venture, G., Ayusawa, K., Nakamura, Y.: Identification of human mass properties from motion. In: IFAC Proceedings Volumes (IFACPapersOnline), vol. 15, pp. 988-993 (2009)

[14] Hansen, C., Venture, G., Rezzoug, N., Gorce, P., Isableu, B.: An individual and dynamic Body Segment Inertial Parameter validation method using ground reaction forces. Journal of biomechanics 47(7), 1577-1581 (2014)

[15] Reinbolt, J.a., Haftka, R.T., Chmielewski, T.L., Fregly, B.J.: Are patientspecific joint and inertial parameters necessary for accurate inverse dynamics analyses of gait? IEEE transactions on biomedical engineering 54(5), $782-793$ (2007)

[16] Zhao, J., Wei, Y., Xia, S., Wang, Z.: Estimating human body segment parameters using motion capture data. 2010 4th International Universal Communication Symposium pp. 243-249 (2010)

[17] Ojeda, J., Martínez-Reina, J., Mayo, J.: The effect of kinematic constraints in the inverse dynamics problem in biomechanics. Multibody System Dynamics (2016)

[18] Babyak, M.A.: What you see may not be what you get: a brief, nontechnical introduction to overfitting in regression-type models. Psychosomatic medicine 66(3), 411-421 (2004)

[19] Pàmies-Vilà, R., Font-Llagunes, J.M., Cuadrado, J., Alonso, F.J.: Analysis of different uncertainties in the inverse dynamic analysis of human gait. Mechanism and Machine Theory 58, 153-164 (2012)

[20] Riemer, R., Hsiao-Wecksler, E.T., Zhang, X.: Uncertainties in inverse dynamics solutions: A comprehensive analysis and an application to gait. Gait and Posture 27(4), 578-588 (2008)

[21] Silva, M.P.T., Ambrósio, J.A.C.: Kinematic Data Consistency in the Inverse Dynamic Analysis of Biomechanical Systems pp. 219-239 (2002)

[22] Wu, G., Siegler, S., Allard, P., Kirtley, C., Leardini, A., Rosenbaum, D., Whittle, M., D'Lima, D.D., Cristofolini, L., Witte, H., Schmid, O., Stokes, I.: ISB recommendation on definitions of joint coordinate system of various joints for the reporting of human joint motion-part I: ankle, hip, and spine. Journal of Biomechanics 35(4), 543-548 (2002)

[23] Wu, G., van der Helm, F.C., (DirkJan) Veeger, H., Makhsous, M., Van Roy, P., Anglin, C., Nagels, J., Karduna, A.R., McQuade, K., Wang, X., Werner, F.W., Buchholz, B.: ISB recommendation on definitions of joint coordinate systems of various joints for the reporting of human joint motion - Part II: shoulder, elbow, wrist and hand. Journal of Biomechanics 38(5), 981-992 (2005)

[24] Featherstone, R.: Rigid Body Dynamics Algorithms (2008) 
[25] Muller, A., Pontonnier, C., Germain, C., Dumont, G.: Dealing with Modularity of Multibody Models. Computer methods in biomechanics and biomedical engineering 18(sup1), 2008-2009 (2015)

[26] Wu, G., Cavanagh, P.R.: ISB recommendations for standardization in the reporting of kinematic data. Journal of biomechanics 28(10), 1257-1261 (1995)

[27] Muller, A., Germain, C., Pontonnier, C., Dumont, G.: A Simple Method to Calibrate Kinematical Invariants: Application to Overhead Throwing. In: International Society of Biomechanics in Sports (ISBS) (2015)

[28] Winter, D.: Biomechanics and motor control of human movement. John Wiley \& Sons (2009)

[29] Damsgaard, M., Rasmussen, J., Christensen, S., Surma, E., de Zee, M.: Analysis of musculoskeletal systems in the AnyBody Modeling System. Simulation Modelling Practice and Theory 14(8), 1100-1111 (2006)

[30] Delp, S., Anderson, F., Arnold, A., Loan, P., Habib, A., John, C., Guendelman, E., Thelen, D.: OpenSim: open-source software to create and analyze dynamic simulations of movement. IEEE transactions on bio-medical engineering 54(11), 1940-50 (2007)

[31] Lu, T.W., O'Connor, J.J.: Bone position estimation from skin marker co-ordinates using global optimisation with joint constraints. Journal of Biomechanics 32(2), 129-134 (1999)

[32] Laitenberger, M., Raison, M., Périé, D., Begon, M.: Refinement of the upper limb joint kinematics and dynamics using a subject-specific closedloop forearm model. Multibody System Dynamics 33(4), 413-438 (2015)

[33] Kuo, A.: A least-squares estimation approach to improving the precision of inverse dynamics computations. Journal of Biomechanical Engineering 120(1), 148-159 (1998)

[34] van den Bogert, A.J., Su, A.: A weighted least squares method for inverse dynamic analysis. Computer methods in biomechanics and biomedical engineering 11(1), 3-9 (2008)

[35] Lund, M.E., Andersen, M.S., de Zee, M., Rasmussen, J.: Scaling of musculoskeletal models from static and dynamic trials. International Biomechanics 2(1), 1-11 (2015)

[36] van der Helm, F.C.: A finite element musculoskeletal model of the shoulder mechanism. Journal of biomechanics 27(5), 551-69 (1994)

[37] de Zee, M., Hansen, L., Wong, C., Rasmussen, J., Simonsen, E.B.: A generic detailed rigid-body lumbar spine model. Journal of biomechanics 40(6), 1219-27 (2007)

[38] Venture, G., Ayusawa, K., Nakamura, Y.: A numerical method for choosing motions with optimal excitation properties for identification of biped dynamics - An application to human. 2009 IEEE International Conference on Robotics and Automation pp. 1226-1231 (2009) 
[39] Cheze, L., Fregly, B., Dimnet, J.: A solidification procedure to facilitate kinematic analyses based on video system data. Journal of Biomechanics 28(7), 879-884 (1995)

[40] Andriacchi, T.P., Alexander, E.J.: Studies of human locomotion: past, present and future. Journal of Biomechanics 33(10), 1217-1224 (2000) 